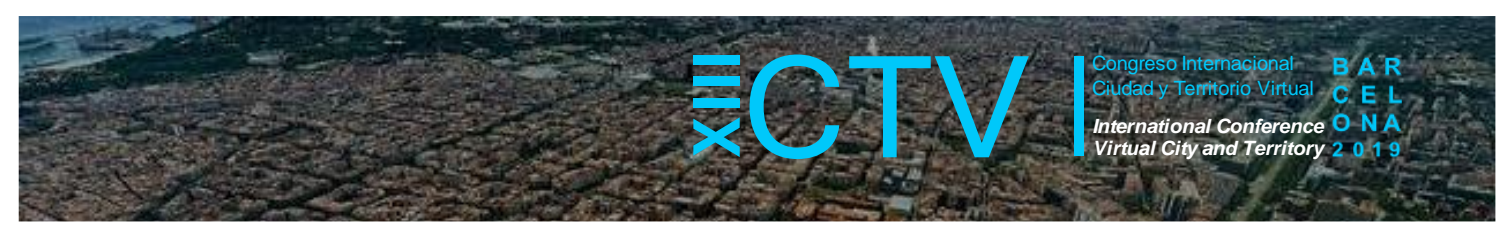

\title{
MOVILIDAD COTIDIANA DE ESTUDIANTES UNIVERSITARIOS. CASO DE LA FACULTAD DE ARQUITECTURA DE LA UNIVERSIDAD CENTRAL DE ECUADOR
}

\author{
Olivares González, Adriana Inés ${ }^{\text {; }}$ Orquera Jácome, María Isabel ${ }^{2}$
}

Remisión inicial: 2019-06-17; Remisión definitiva: 2019-10-20; Publicación: 2019-12-21

Citación: Olivares-González, A. \& Orquera-Jácome I. (2019). Movilidad cotidiana de estudiantes universitarios. Caso de la Facultad de Arquitectura de la Universidad Central de Ecuador. En XIII CTV 2019 Proceedings: XIII International Conference on Virtual City and Territory: "Challenges and paradigms of the contemporary city”: UPC, Barcelona, October 2-4, 2019. Barcelona: CPSV, 2019, p. 8692. E-ISSN 2604-6512. DOI http://dx.doi.org/10.5821/ctv.8692

\section{Resumen}

Uno de los temas que se encuentran de manera permanente y creciente en la agenda de los gobiernos metropolitanos y locales de las ciudades latinoamericanas es la movilidad urbana, por sus implicaciones tanto en la eficiencia, productividad y competitividad de la ciudad (WBCSD, 2013; ONU-Habitat, 2015) como en la calidad de vida de sus habitantes, sobretodo, en lo relativo a la salud y el tiempo libre (Miralles, 2010).

En particular Quito, capital de Ecuador, alberga un conjunto de variables que han dificultado la gestión de la movilidad urbana y esto afecta a todos los habitantes, sobretodo, en sus desplazamientos ocupacionales -trabajo y escuela-. Para esta reflexión nos centraremos en la problemática que tienen los estudiantes universitarios para desplazarse, tomando como caso la Facultad de Arquitectura de la Universidad Central de Ecuador (UCE) que, como la universidad pública de país, aglomera a más del $30 \%$ de estudiantes de la provincia de Pichincha, es decir, 47.700 estudiantes.

La problemática se sitúa en la localización de la Universidad Central de Ecuador y su Facultad de Arquitectura, en el denominado Hipercentro de la ciudad, que aglutina además a las 12 universidades con las que cuenta el Distrito Metropolitano de Quito (DMQ) y a las que se desplazan diariamente cerca de 159 mil estudiantes.

Además de las universidades, se concentran en el Hipercentro otras actividades económicas y equipamientos urbanos que generan diariamente el $60 \%$ de los desplazamientos en transporte público y el $40 \%$ de los desplazamientos en trasporte privado (DMQ, 2014). Por otro lado, en términos territoriales, Quito cuenta con una morfología de ciudad lineal de $42 \mathrm{~km}$ de largo y solo $4 \mathrm{~km}$ de ancho (Cuenin \& Silva, 2010), que se extiende hacia el norte y el sur, de manera que la limitada red vial que corre en ese sentido y la gran cantidad de usuarios que se desplazan generan problemas constantes de tráfico y congestión. Las infraestructuras de transporte público del DMQ están formadas por tres ejes: la Ecovía, el Trolebús, el Metrobús y próximamente el Metro de Quito, y todas enlazan a la ciudad en el mismo sentido.

En este contexto, el objetivo de este trabajo es analizar que tanto la morfología urbana, la infraestructura de movilidad y la localización de la UCE; afecta a los estudiantes de diferentes estratos socioeconómicos de la facultad de arquitectura en sus desplazamientos cotidianos, el cual corresponde a una parte del trabajo de tesis doctoral denominada Estilos de vida y movilidad cotidiana en los estudiantes universitarios. Caso Facultad de Arquitectura y Urbanismo en el Distrito Metropolitano de Quito (DMQ), coautora de este documento.

La metodología parte del análisis de los itinerarios cotidianos de 41 estudiantes de la Facultad de Arquitectura de la UCE, mediante un GPS integrado a la aplicación Wikiloc, elegida por la facilidad de instalación en los celulares dichos estudiantes y por ser una aplicación con la que están familiarizados. Mediante una entrevista a cada estudiante se identificaron la frecuencia de cada desplazamiento y los motivos divididos por actividades de estudios universitarios, deportivas, laborales, salud, alimentación, familiares, abasto y recreación. Esta información a su vez se cruzó con la localización de la residencia del estudiante y el nivel socioeconómico que dicha localización representa, considerando los tiempos de viaje.

\footnotetext{
${ }^{1}$ Universidad de Guadalajara, Instituto de Investigación y Estudios de las Ciudades, México. http://orcid.org/0000-00027391-0684, Correo de contacto: ines.olivares@académicos.udg.mx; ${ }^{2}$ Universidad Central de Ecuador, Facultad de Arquitectura y Urbanismo, Quito, Ecuador, https://orcid.org/0000-0002-0165-3172, Correo de contacto: miorquera@uce.edu.ec
} 


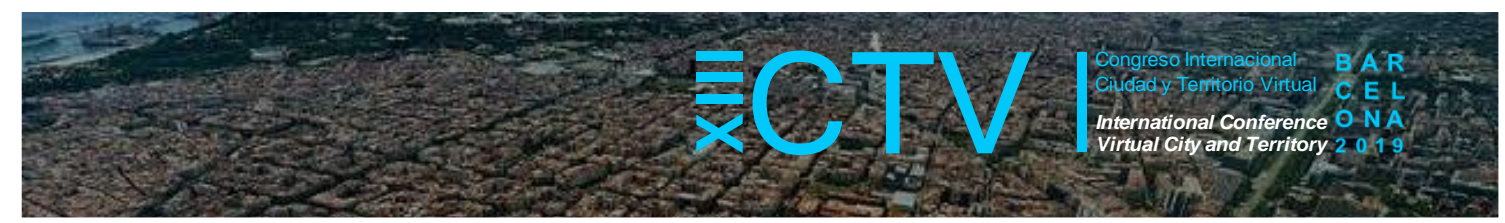

Entre los resultados, se identificó que los estudiantes que habitan en los límites urbanos norte y sur del DMQ, que cuentan con menor accesibilidad urbana, son los que emplean mayor tiempo en sus desplazamientos ocupacionales, con independencia de su nivel socioeconómico. Asimismo, el nivel de diversidad funcional de la localización residencial de los estudiantes, que caracteriza positivamente al Hipercentro del DMQ y negativamente a las colonias externas del mismo, influyen en el mismo sentido, en la variedad y frecuencia de sus actividades cotidianas.

Por lo tanto, comprobamos que la accesibilidad y la diversidad funcional de los lugares de residencia de los estudiantes universitarios son fundamentales para el desarrollo de actividades que promueven su desarrollo integral, ya que se promueve una mayor frecuencia de actividades de salud y de recreación; así como el cuidado de su alimentación. Por otro lado, resalta que las actividades deportivas no están relacionadas con la accesibilidad a equipamientos especializados, sino con otros factores de carácter cultural en los que seria necesario profundizar. Asimismo, se constató que, si hay un componente de género en la organización de los desplazamientos de los estudiantes con hijos, influenciados por su papel como responsables de estos y del hogar.

\section{Abstract}

One of the issues that are permanently and increasingly on the metropolitan and local governments of Latin America agenda is urban mobility. This is due to its implications on efficiency, productivity and competitiveness of the city (WBCSD, 2013; UN -Habitat, 2015) as well as in the quality of life of its inhabitants, especially in relation to health and leisure (Miralles, 2010).

In particular, Quito, the capital of Ecuador, has a set of variables that have hindered the management of urban mobility. This has affected all inhabitants, especially in their commute to work and school-. On this paper we will focus on the problem that university students have to commute. For this investigation the Faculty of Architecture of the Universidad Central Del Ecuador (UCE) has been taken as a case study. Central University Universidad Central Del Ecuador brings together more than $30 \%$ of students from Pichincha province, around 47,700 students.

The problem is generated because of the location of Universidad Central Del Ecuador and its Faculty of Architecture. It is located in the city's Hipercentro, which houses the twelve universities that the Metropolitan District of Quito (DMQ) has, and where daily 159 thousand students commute to.

In addition to universities, there are businesses and urban facilities, where multitudes of citizen's have to commute, generating $60 \%$ of public transportation and $40 \%$ of private transportation journeys (DMQ, 2014). On the other hand, in territorial terms, Quito has a linear city morphology of $42 \mathrm{~km}$ long and only $4 \mathrm{~km}$ wide (Cuenin \& Silva, 2010), which extends from north to south. Therefore, Quito has a limited road network generating constant traffic and congestion problems. The DMQ public transport infrastructure consists of three axes: The Ecovía, the Trolebus, the Metrobús and soon Quito's subway, these three transportation systems are interconnected to transport Quito's cities' from north to south.

In that context, the objective of this paper is to analyze that urban morphology, mobility infrastructure and the location of the UCE; affect students from different socioeconomic status of the faculty of architecture in their everyday displacement. These case study corresponds to a part of the doctoral thesis work called Lifestyles and everyday mobility in university students. Case of the School of Architecture and Urbanism in the Metropolitan District of Quito (DMQ), from one of the co-authors of this document.

The methodology starts from the analysis of the daily itineraries of 41 students of the Faculty of Architecture of the UCE, through an integrated GPS app called Wikiloc, chosen for its easy installation on cell phones and for being an application with which students are familiar with. Through an interview with Each student, the frequency of each trip and the motives which were divided by activities of university studies, sports, work, health, food, family, supplies and recreation were identify. This information was crossed with the location of the student's residence and the location's socioeconomic level, taking into account their everyday mobility.

Among the results, it was identified that students who live in the northern and southern urban limits of the DMQ, and have less urban accessibility, are those who spend more time commuting, regardless of their socioeconomic status. Likewise, diversity of activities and the residential location of the students, affects positively the Hipercentro of the DMQ and negatively to the external neighborhoods, These aspects influence in the same sense, in the variety and frequency of their daily activities.

Therefore, we verify that the accessibility and functional diversity of the places of residence of university students are fundamental for the development of activities that promote their development. This will promote a greater frequency of health and recreational activities; as well as taking care of their eating habits. On the other hand, it emphasizes that 


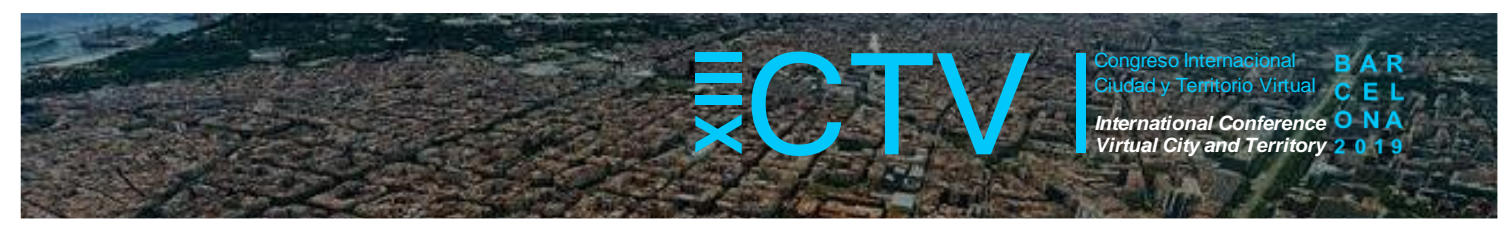

sports activities are not related to accessibility to special urban facilities, but to other cultural factors in which it would be necessary have further studies. It was also found that if there is a gender component in the organization of students' displacements with children, influenced by their role as resp for them and the home.

Palabras Clave: movilidad cotidiana, estudiantes universitarios, ciudad lineal, sostenibilidad

Key words: everyday mobility, university students, linear city, sustainability

\section{Quito, Ecuador: morfología e infraestructuras para la movilidad}

Ecuador, o República del Ecuador, se encuentra ubicado en el sur del Continente Americano, está atravesado por la línea ecuatorial a la que debe su nombre. San Francisco de Quito, más conocida como Quito, es la capital del Ecuador y de la provincia de Pichincha, además pertenece al único distrito del país, el Distrito Metropolitano de Quito (DMQ).

El DMQ se encuentra asentado en la hoya de Quito que es un territorio localizado en medio de las Cordillera Occidental y Oriental de los Andes y forma la cuenca alta del Río Guayllabamba (IMQ Dirección de Planificación, 1992), en la parte oriental se ubican las elevaciones del Casitagua, Pichincha y Atacazo que constituyen una verdadera barrera pues superan los 4.000 m.s.n.m. y son parte de la zona de protección ecológica; mientras que al este, la muralla de la cordillera oriental quiebra la continuidad del valle de Quito con los valles de Cumbayá, Tumbaco y Los Chillos (D’Ercole \& Metzger, 2004).

EI DMQ no sólo tiene como inconveniente las cordilleras ya mencionadas sino también una fragmentación espacial derivada de diferentes obstáculos orográficos, entre los que destacan la loma del Panecillo, elevación de 3.000 m.s.n.m. ubicada en el centro geográfico de la ciudad y cerca del Centro Histórico; el volcán llaló con 3.188 m.s.n.m. que divide el valle de Cumbayá y Tumbaco con el valle de los Chillos; así como otros obstáculos hidrográficos (río Machángara, San Pedro, Chiche, Guayllabamba, etc.). La difícil condición topográfica de la ciudad es acentuada por ciertos espacios urbanos que por su dimensión se constituyen en barreras a la accesibilidad urbana, tales como el parque Bicentenario (antiguo aeropuerto de la capital) con $3.8 \mathrm{kms}$ de largo, el parque la Carolina con una longitud de $1.6 \mathrm{~km}$ y el Centro Histórico cuyo acceso vehicular es restringido por su declaratoria de Patrimonio de la Humanidad; los cuales en conjunto constituyen obstáculos mencionados para la conectividad de este a oeste (D’Ercole \& Metzger, 2004).

La ciudad se asentó en el valle de Quito que comprende una buena parte del altiplano con algunos sectores parcialmente accidentados con pendientes que fluctúan de los 2.700 a 2.900 m.s.n.m. (IMQ Dirección de Planificación, 1992). La expansión de la ciudad, por las razones topografías y antrópicas mencionadas, se organizó de norte a sur mediante una morfología lineal que para 2010 era de $42 \mathrm{~km}$ de largo y solo $4 \mathrm{~km}$ de ancho en su lado más angosto (Cuenin \& Silva, 2010). Las cordilleras y los obstáculos ya mencionados, sumados a los marcados desniveles y el crecimiento longitudinal de la ciudad, han provocado complicaciones en los desplazamientos que se realizan cotidianamente tanto dentro del DMQ como hacia las afueras del mismo, tal es así que para ingresar a la capital desde los valles orientales, hay que ascender las vertientes que rodean la ciudad antes de poder descender hacia el espacio central, donde se encuentran algunos tramos viales con pendientes relativamente fuertes que dificultan la conducción. Esta condición implica asimismo un alargamiento significativo de las distancias a la vez que un incremento de los tiempos de los desplazamientos (D'Ercole \& Metzger, 2004). 


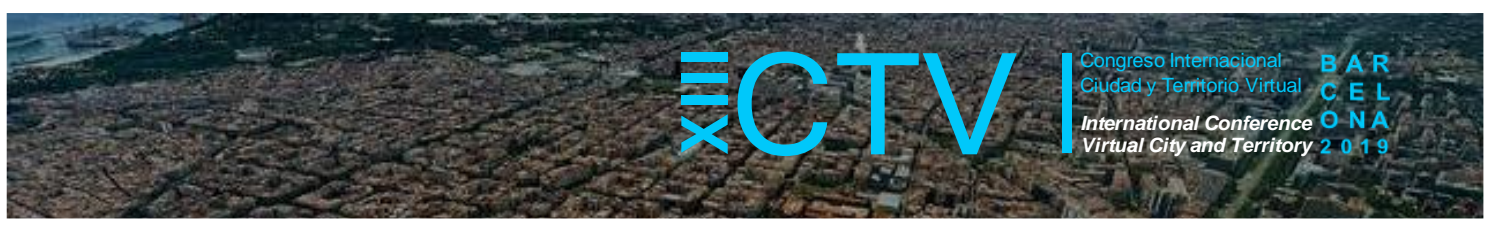

En el diagnóstico que la administración metropolitana hace en el informe Quito, Visión 2040, destaca que "la geografía del DMQ es una condicionante para la conformación de una red vial urbana compacta y ordenada" (IMPU, 2018 pág. 118.), ya que condiciona la conectividad urbana, el número y continuidad de rutas alternativas, las distancias entre puntos de origendestino, así como el uso de modos no motorizados. A esto se suma la baja calidad física de las vías y su prioridad para el uso del automóvil.

El escaso espacio de reserva urbana impide la creación de nuevas vías y, cuando se implementan, afectan otras áreas habitacionales, mediante la fragmentación del tejido urbano y social, y/o el desplazamiento de los residentes, generalmente de bajos recursos, todo esto sin considerar el transporte público como alternativa para la movilidad urbana (IMPU, 2018).

La distribución modal de los viajes diarios en el DMQ, según datos de 2017, era dominado por los modos motorizados (IMPU, 2018, pág. 210). El transporte público esta formado por el Sistema Integrado de Transporte (SIT) que cuenta con 5 corredores de transporte masivo, mediante sistemas Bus Rapid Transit (BRT), dispuestos en el eje longitudinal de la ciudad como se muestra en Figura 1; así como rutas de transporte colectivo, que utilizan autobuses que emiten gases contaminantes, los conductores irrespetan las señales de tránsito y no ofrecen un buen trato a los usuarios. El Metro de Quito, en proceso de construcción, su sumará a los medios masivos en el año 2020, según comunicaciones oficiales.

EI SIT funciona deficiente, pues no cuenta con información de horarios ni frecuencia de paso. No todos los corredores de transporte tienen atención nocturna y los que, si la tienen, la tarifa es el doble del costo normal, el confort de los usuarios se ve afectado por el mal estado de la superficie de la capa de rodadura del carril exclusivo del BRT y por la calidad del mobiliario (IMPU, 2018). En conclusión, la movilidad sostenible que tiene entre sus atributos sociales la accesibilidad, asequibilidad, confiabilidad y la equidad, sigue siendo un desafío para el DMQ.

Figura 1. Mapa de la estructura orográfica, red vial primaria, Sistema Integrado de Transporte, universidades del Distrito Metropolitano de Quito, Ecuador y del Hipercentro del DMQ

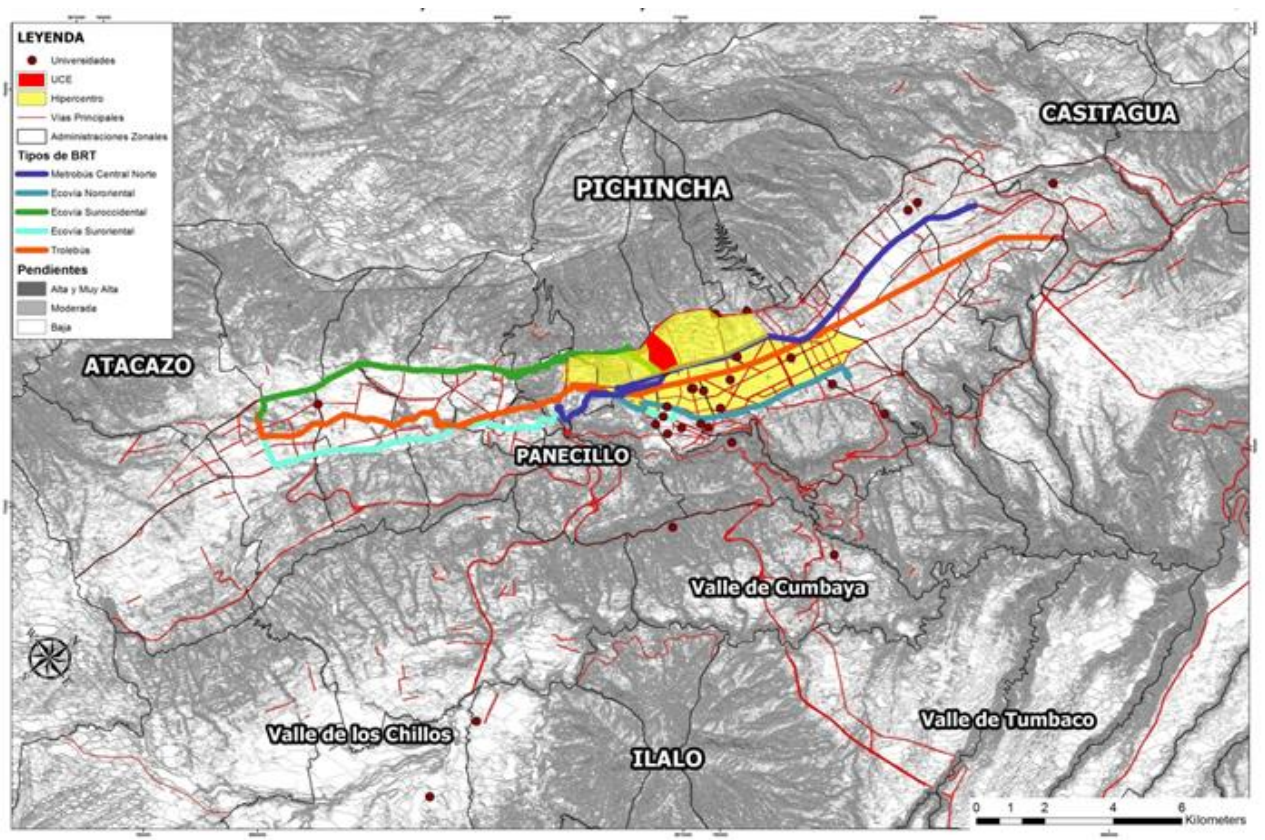

Fuente: Elaboración propia con base información de la Secretaría de Movilidad del DMQ 


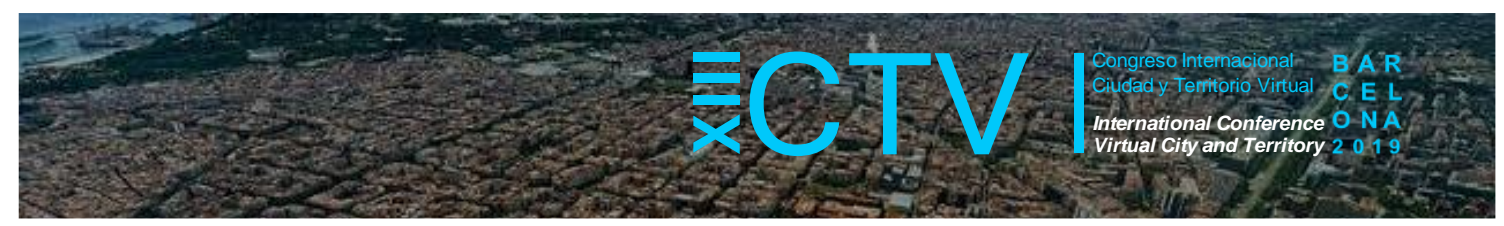

\section{La Universidad Central de Ecuador y el Hipercentro del Distrito Metropolitano de Quito}

La topografía del territorio donde se emplaza el $D M Q$, ha delineado la formación de una ciudad alargada, fragmentada longitudinalmente por arterias principales y separada de los valles al Oriente, por el marcado desnivel topográfico, como se puede observar en la Figura 1.

Por otro lado, desde los años 20, con el aparecimiento de los primeros barrios modernos de la ciudad, hacia el norte la Mariscal y América y, al sur la Magdalena, la ciudad pasó de un modelo compacto y mixto, hacia uno disperso y fragmentado con una escasa consideración de las condiciones geográficas del territorio para su crecimiento, lo que ha dado como resultado deficiencias funcionales y riesgos ambientales: el modelo de vivienda actual está impulsado por enclaves privados con vías de acceso exclusivo, desconectados del trazado urbano, existiendo vías privadas y/o sin salida; hay una sobre-concentración de equipamientos y actividades económicas en el denominado Hipercentro a la vez de una disminución de su densidad poblacional, mientras que la red vial transversal y de conexión con los valles es insuficiente; en términos ambientales los asentamientos han ido escalando las laderas y hay diversos riesgos por deslizamientos causados por lluvias o sismos.

El DMQ con una población aproximada de dos millones de habitantes (según datos censales del 2010), genera un total de 4'600.000 viajes diarios (MDDM de Quito, 2014). El Hipercentro, como ya se mencionó, es la zona donde se agrupa la mayor cantidad de equipamientos urbanos, públicos y privados, así como fuentes de trabajo, es la zona que mayor atracción de viajes genera en el DMQ, aproximadamente el 40\% (313.494 viajes diarios) del total de viajes atraídos son en transporte privado y el 60\% (652.418 viajes diarios) de los viajes atraídos son en transporte público (MDDM de Quito, 2014). Al Hipercentro acuden no sólo personas que habitan en el DMQ sino también personas de ciudades y poblaciones aledañas al distrito, a medida que el usuario se aproxima al Hipercentro, puede evidenciar como se va complicando la movilidad.

Los habitantes de Quito viven cada vez más lejos de sus principales destinos de viaje, sus actividades están concentradas principalmente en el Hipercentro y a las que acceden de manera ineficiente a través de una red vial congestionada con un rango extremadamente limitado de opciones para realizar sus traslados cotidianos (IMPU, 2018). Es en el DMQ, donde se ubica el mayor número de Universidades del país, por ende, es el territorio con el mayor número de estudiantes universitarios registrados. Según datos de la Secretaría de Educación Superior, Ciencia, Tecnología e Innovación (SENECYT), son 21 universidades cuyos campus están en la provincia de Pichincha, de las cuales 7 son universidades públicas y 14 universidades privadas. De las 21 universidades, 17 se ubican dentro o de forma lindante al polígono del Hipercentro del DMQ. Las 17 universidades en mención suman aproximadamente 130 mil estudiantes (SENESCYT, 2017), lo que quiere decir que son 130 mil viajes diarios obligatorios cuyo único motivo es el estudio en la universidad. Este sector recibe una gran afluencia diaria (de lunes a viernes) de estudiantes, que constituyen desplazamientos obligatorios y que también son conocidos como movilidad ocupacional. Con lo mencionado, se puede afirmar que el DMQ cuenta con una Centralidad Universitaria por la ubicación geográfica y cercanía que existe entre estas Instituciones de Educación Superior, en la Figura 1 se puede apreciar la ubicación de las universidades en el DMQ, recalcando que existen universidades que tienen más de un campus. 


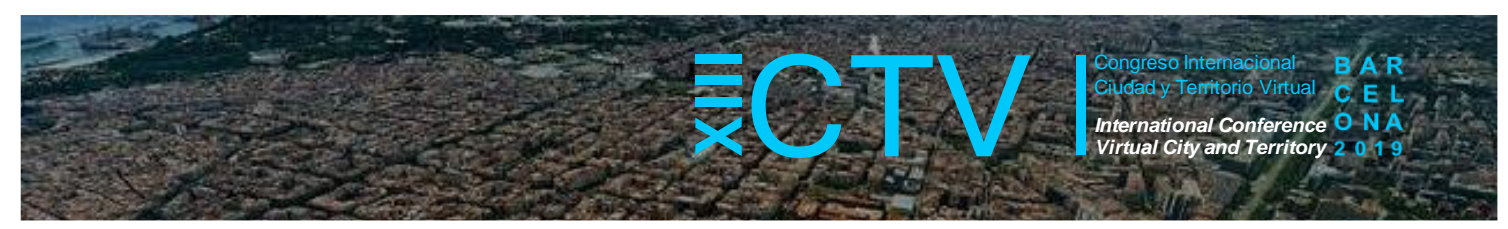

La Universidad Central del Ecuador (UCE), universidad pública con mayor trayectoria y la más antigua del país (fundada en 1620), es la institución que alberga a 47.706 alumnos (27.598 mujeres y 20.108 hombres), de los cuales aproximadamente el $30 \%$ provienen del DMQ (SENESCYT, 2017) y el resto de todas las provincias del país, inclusive de otros países, por la variedad de carreras de pregrado que oferta (57 carreras) en sus 21 Facultades. Los estudiantes de la Facultad de Arquitectura y Urbanismo de la UCE (FAU-UCE) son el sujeto de estudio, los cuales suman 1476 estudiantes matriculados en 2018 (46\% mujeres y 54\% hombres), según información obtenida de la Dirección de Carrera de Arquitectura FAU-UCE.

\section{Metodología y levantamiento de información}

Existen varios estudios de diferentes épocas que analizan la movilidad en el $D M Q$, tales como Estructura espacial Metropolitana: Temas y Problemas críticos (1992) y el Diagnóstico de la movilidad en el DMQ para el Plan Metropolitano de Desarrollo Territorial (2014) entre otros, sin embargo estos se concentran en recabar información de origen-destino de los desplazamientos, por ello se considera relevante la necesidad de hacer estudios más detallados que permitan entender la movilidad desde las características y elecciones de los individuos. El método del tracking es relevante para los estudios detallados de movilidad ya que permite geo-referenciar la trayectoria y el tiempo real de los desplazamientos, lo cual es relevante en consideración de que en los estudios tradicionales se registra la información que recuerdan y perciben los sujetos, que e muchos casos es poco cercana a la realidad.

Esta investigación corresponde a una parte del trabajo de tesis doctoral denominada Estilos de vida y movilidad cotidiana en los estudiantes universitarios. Caso Facultad de Arquitectura y Urbanismo en el Distrito Metropolitano de Quito (DMQ), que dirigen y desarrollan respectivamente las coautoras de este documento. El sujeto de estudio son los estudiantes de la FAU-UCE, como ya se mencionó, ya que su experiencia de movilidad es significativa por estar entre los grupos de concurrencia cotidiana al DMQ. Para el tracking se convocó la participación de los estudiantes por medio de los programas de prácticas pre-profesionales y vinculación con la sociedad, que son requisito establecido en la Ley Orgánica de Educación Superior (LOES) para la titulación de todos los estudiantes universitarios en el Ecuador, ya que se requería garantizar su participación comprometida en las actividades que requería el experimento. Participó un grupo voluntario de 46 estudiantes de entre cuarto y séptimo semestre de diez que integran la licenciatura, con edades de entre 20 y 23 años. Una vez revisada la calidad de la información se seleccionaron los casos de 41 estudiantes de los cuales 23 son mujeres y 18 son hombres, 14 proceden de otras provincias y viven solos o con familiares y 27 proceden del DMQ. Ya que Ecuador no tiene ley para protección de datos personales se elaboró un documento donde se establecen límites en el manejo de la información para dar seguridad a los estudiantes.

Las variables que se consideraron fueron las siguientes: lugar de residencia de los estudiantes, que en la mayoría de los casos es el punto de origen del desplazamiento hacia la UCE; motivos y modos de los desplazamientos; y finalmente su procedencia (DMQ u otras provincias) y su estrato social. Previo a la aplicación de los instrumentos, se realizaron reuniones de trabajo y capacitación a los estudiantes, para garantizar la calidad de la información. Para poder comprender la movilidad cotidiana, y obtener información de las tres primeras variables se levantaron los itinerarios cotidianos de los 46 estudiantes por un periodo de 21 días, por medio 


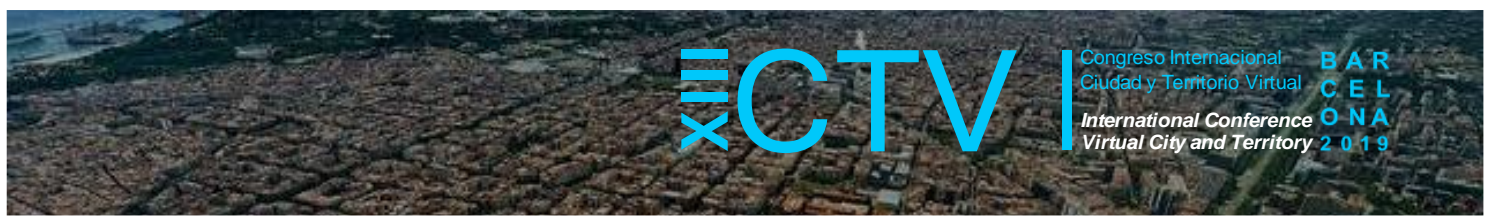

de dos instrumentos: la georeferenciación de los desplazamientos cotidianos por medio de GPS y una bitácora diaria.

La aplicación Wikiloc ${ }^{2}$ fue seleccionada para la geo-referenciar los desplazamientos diarios, por la facilidad de instalación en los celulares y la familiaridad que los estudiantes tenían con su uso. La bitácoras cotidianas se diseñaron en formato Excel, en donde el estudiante registró, de manera vertical cada desplazamiento y, de forma horizontal, sus características: fecha de desplazamiento (día, mes, año), lugar de inicio del desplazamiento (calle principal, calle secundaria), lugar de llegada (calle principal, calle secundaria), tiempo de recorrido (tiempo en minutos para saber la duración del desplazamiento), horario de salida (hora y minuto de inicio de la ruta), hora de llegada (hora y minuto de finalización de la ruta), motivo (considerando estilos de vida saludables: estudios universitarios, deportes, trabajo, salud, abasto, capacitaciones, alimentación, visitas familiares y retorno al lugar de residencia) y modo (A pie, bicicleta, transporte público, transporte privado).

Una vez instalada la aplicación, los alumnos comenzaron a levantar registrar sus los desplazamientos y al finalizar cada día descargaban el recorrido y llenaban la bitácora que enviaban por correo electrónico. Por la calidad de la información fueron descartados 5 casos de estudiantes que no cumplieron con los requisitos que se solicitaron.

En lo que se refiere al estrato social, dada la ausencia de información actualizada, se calculó a partir de los siguientes criterios sociales y económicos: ocupación, ingreso económico, características de la vivienda y acceso a servicios básicos con los que se establecieron estratos sociales a nivel de zona censal del $\mathrm{DMQ}^{3}$. Esta información a su vez se cruzó con la localización de la residencia del estudiante definir su estrato social.

Toda la información de los dos instrumentos de levantamiento se procesó en Sistemas de Información Geográfica y para el análisis de resultados se elaboró una tabla resumen que contiene el tiempo de viaje promedio de los desplazamientos realizados por los diferentes motivos, organizados en un umbral de hasta 20 minutos y 5 mapas que muestran la estructura típica de los desplazamientos por modo.

\section{Influencia del modelo lineal de la morfología e infraestructuras de transporte y la hiper concentración de universidades en la movilidad de los estudiantes}

La localización residencial y el estrato socio-económico de los estudiantes de la UCE definen el modo y el tiempo de sus desplazamientos cotidianos como podemos observar en el resumen de resultados de la Figura 2 y Figura 3.

\footnotetext{
${ }^{2}$ Wikiloc: es una aplicacion que dispone mapas gratuitos y una plataforma de información geográfica voluntaria para descubrir y compartir información. (Wikiloc)

${ }^{3}$ Son áreas que agrupan sectores censales, con menor extensión de empadronamiento, que poseen un tamaño similar tanto en cantidad de población como en superficie territorial. (Guía para la elaboración de un proyecto censal, CEPAL, 2010)
} 


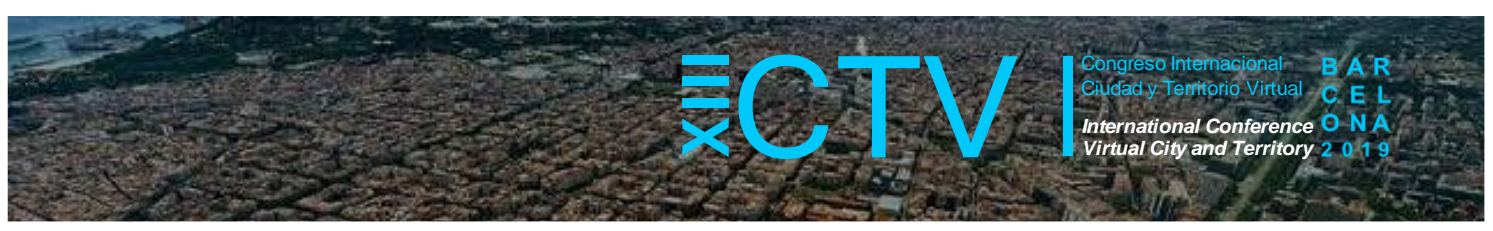

Figura 2. Número de desplazamientos según motivo

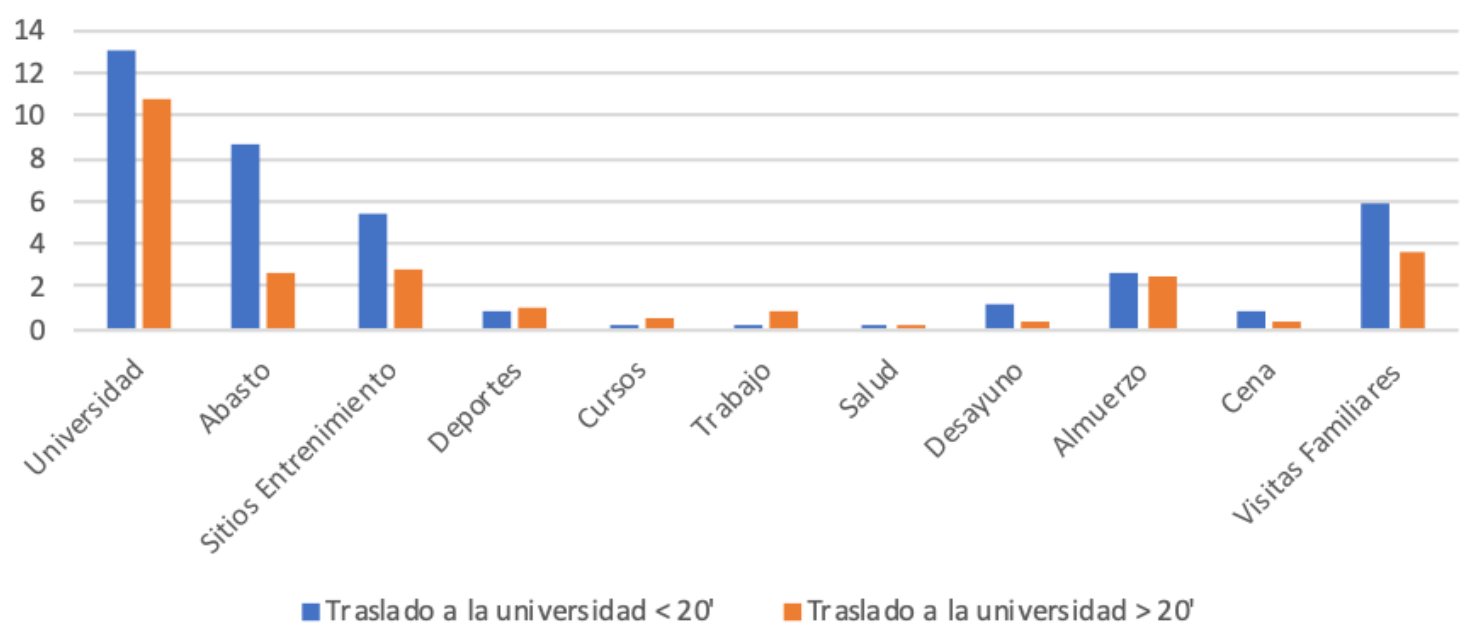

Fuente: Levantamiento de rutas cotidianas de estudiantes de la UCE-FAU

Figura 3. Tiempo de desplazamiento promedio según el motivo

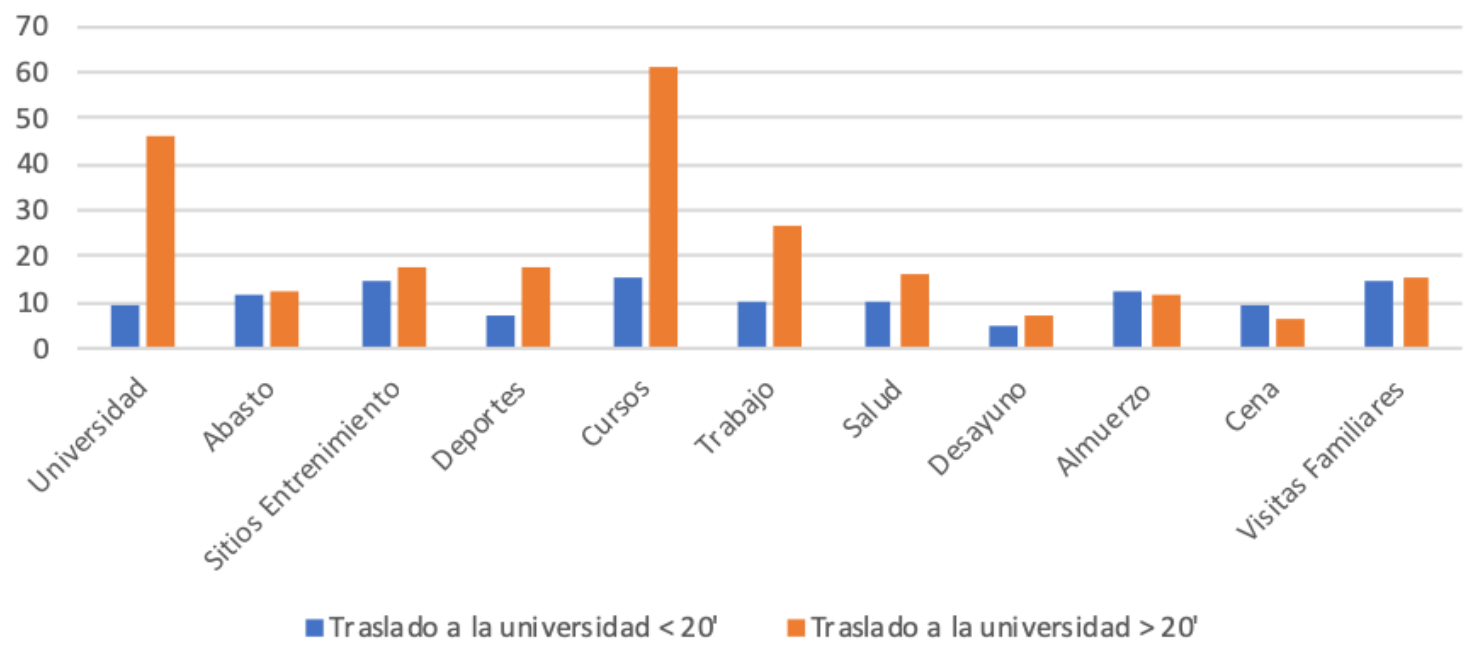

Fuente: Levantamiento de rutas cotidianas de estudiantes de la UCE-FAU

Los estudiantes que residen en los alrededores de la Facultad de Arquitectura se desplazan a pie, sin embargo el umbral para hacer dicha elección modal es de 20 minutos que está en el rango máximo de tiempo de los desplazamientos peatonales, supera con mucho el tiempo óptimo establecido por la literatura para los desplazamientos de proximidad que es de 10 minutos (Ryley 2008, Miralles-Guash, 2008, 2011, 2012; Marquet, O., \& Miralles-Guasch, C.,2014) y también el tiempo promedio de los desplazamientos a pie para las 10 ciudades de América Latina que es de 15 minutos (Observatorio de Movilidad Urbana de América Latina, CAF, 2007-2011), esto se debe muy posiblemente a la edad de los estudiantes que como establecimos está en el rango de entre los 20 y 23 años. Por otro lado, los estudiantes que residen alrededor de la UCE, ya que la UCE se localiza en el Hipercentro del DMQ, los alumnos que se desplazan a pie tienen acceso a una amplia diversidad de equipamientos y servicios que influyen también en el modo y la frecuencia con la que realizan la mayor parte de sus actividades cotidianas restantes: abasto, entretenimiento y visitas familiares. 


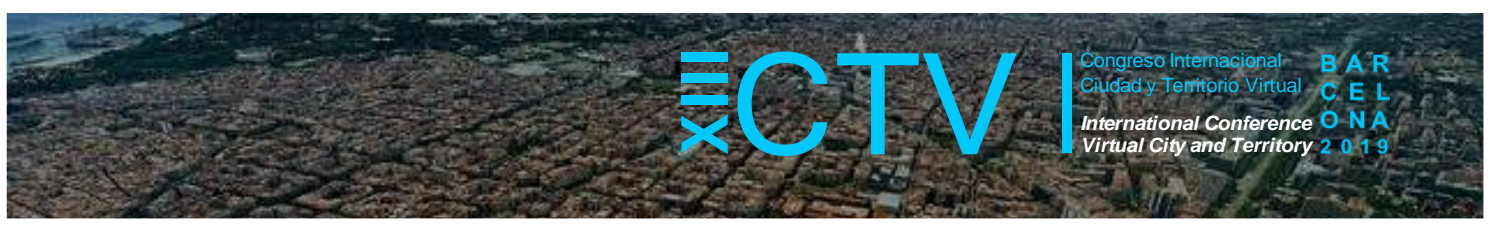

En la Figura 4 se puede observar un día cotidiano de un estudiante que vive cerca al predio universitario, su desplazamiento a la UCE lo realiza a pie y todas sus actividades las desarrolla en torno a su lugar de su residencia, en la figura se aprecia que ese día realizó un trayecto más prolongado hacia el Centro Histórico de Quito, al sur de la UCE, cuyo motivo fue la distracción y el tomar fotografías para un trabajo académico.

Figura 4. Mapa de un día cotidiano de desplazamiento a pie a la FAU

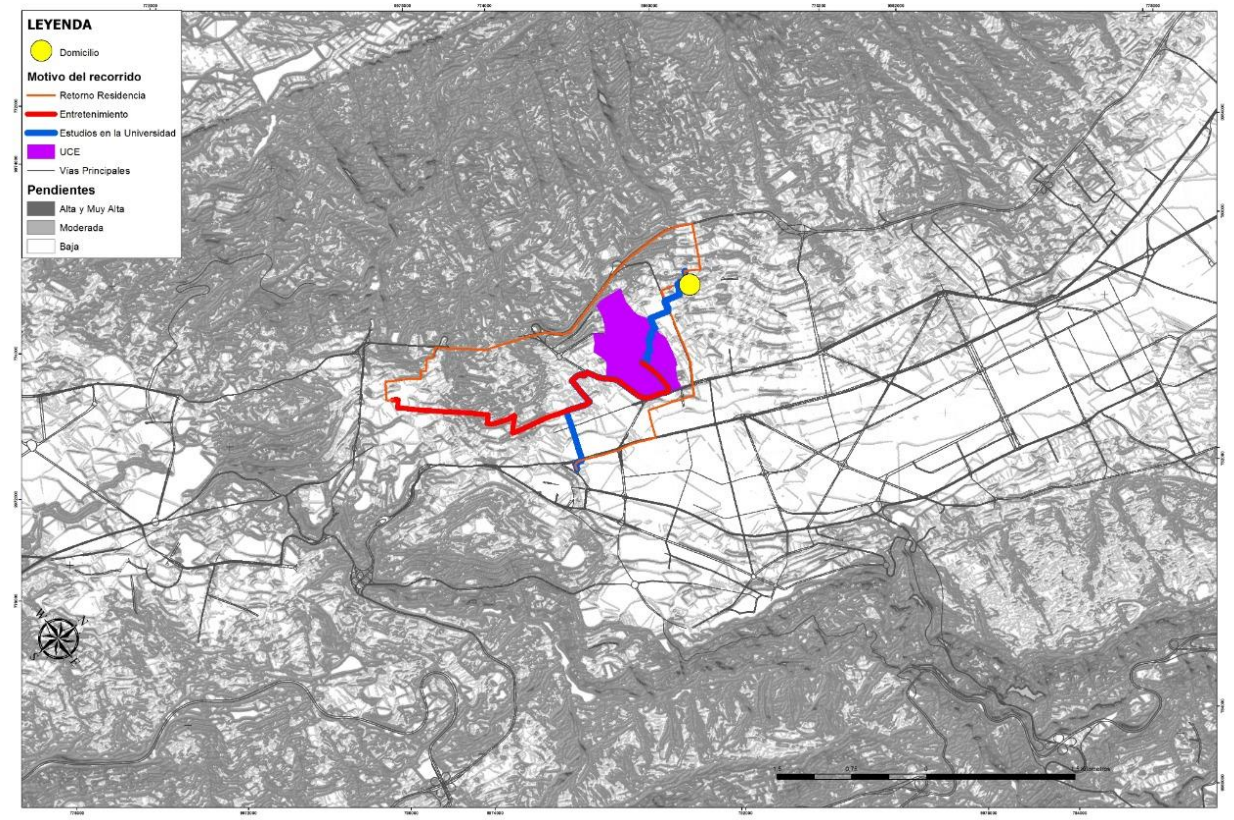

Fuente: Levantamiento de rutas cotidianas de estudiantes de la UCE-FAU

La mayoría de los estudiantes que viven cerca de la universidad (6 de 8) proceden de otras provincias, de manera que viven en alquiler. No obstante, los prolongados tiempos de viaje para visitar a su familia cercana, estos estudiantes no descuidan el contacto familiar, de manera que los que tardan en promedio 2 horas realizan visitas semanales o quincenales mientras que los que viven hasta a 10 horas de tiempo, sus visitas tienen por lo general una frecuencia mensual.

De manera que el bajo tiempo del desplazamiento cotidiano también influye en un estilo de vida más activo en cuanto a actividades recreativas y familiares, incluso en aquellos que viven en otras provincias. Este grupo de población corresponde al $15.7 \%$ de los estudiantes de la UCE ${ }^{4}$ mientras que en la muestra corresponde al $20 \%$.

El $71.8 \%$ de los estudiantes se traslada en transporte público (Figura 5), es decir, casi tres cuartas partes del total de la FAU. Este tipo de desplazamientos escolares son los que consumen el mayor tiempo de tiempo con un rango de entre 50 y 76 minutos, superando el tiempo óptimo establecido por organismos internacionales para este tipo de transporte que es de 30 minutos (ONU-Habitat, 2016). La asequibilidad es la razón principal por la que los estudiantes se desplazan en esta modalidad, ya que el costo de los billetes asciende a 25 centavos de dólar y con ese valor se puede cruzar la ciudad $(42 \mathrm{~km})$ de norte a sur,

\footnotetext{
${ }^{4}$ En Censo fue elaborado por Isabel Orquera Jácome, coautora de este documento con motivo de su tesis de doctorado, entre el 26 y 30 de marzo de 2018
} 


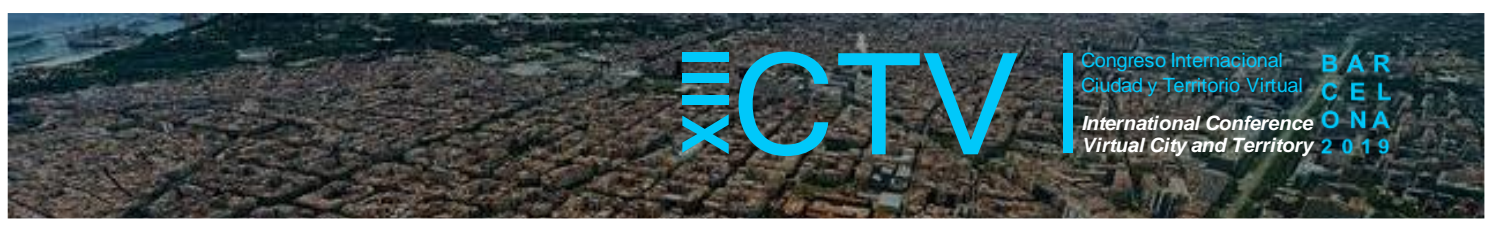

atravesando el Hipercentro. Estos estudiantes por lo general utilizan dos unidades de transporte público por lo que el costo del viaje no supera los 50 centavos de dólar, sin embargo, hay casos en los que utilizan hasta 3 unidades de transporte público.

Los tiempos de desplazamiento en transporte público son superiores a los que se realizan en transporte privado, a pesar de que las líneas de transporte masivo tienen carriles exclusivos, lo que evidencia los largos tiempos de espera, es decir, la poca frecuencia de las unidades del transporte masivo. El lugar de residencia de los estudiantes que utilizan el transporte público, generalmente esta fuera del Hipercentro en colonias de nivel socio-económico medio, por lo que la localización residencial influenciada por la capacidad económica si influye en el uso del transporte público y en la frecuencia de otras actividades no escolares, como las recreativas.

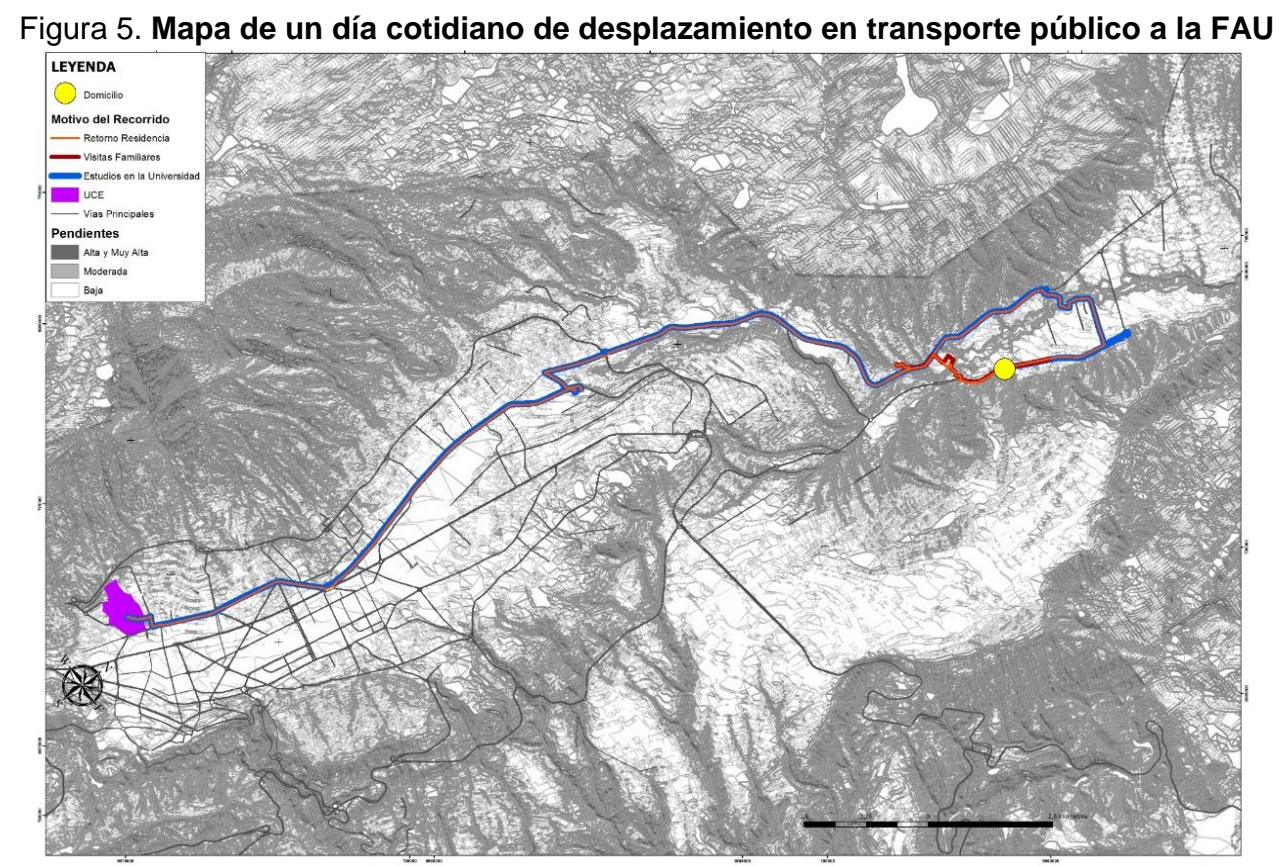

Fuente: Levantamiento de rutas cotidianas de estudiantes de la UCE-FAU

Solo el $4.8 \%$ de los estudiantes de la FAU realizan sus desplazamientos en transporte privado, aunque en la muestra fueron casi la mitad de los voluntarios (41\%). Todos residen en el DMQ, la mayoría fuera del Hipercentro, y viven con sus familiares en colonias de nivel medio-alto de manera que el auto es generalmente propio (Figura 6).

Solo la mitad de la muestra tiene tiempos de desplazamiento de entre 20 y 30 minutos, el máximo establecido en la literatura para transporte privado (ONU-Habitat, 2016) y la otra mitad hace de 46 a 60 minutos; de manera que la capacidad económica de los estudiantes no es una condición que garantice menores tiempos de desplazamiento, situación que a su vez incide en la frecuencia de otras actividades cotidianas como las recreativas. Por otro lado, ya que las zonas donde residen estos estudiantes están menos servidas en equipamientos deportivos y de salud, para dichos traslados utilizan el transporte privado, sin embargo, se observa su excesivo uso para realizar las actividades de alimentación fuera de casa (desayuno, cena) donde sus tiempos de desplazamiento son apenas de 2 a 5 minutos. Hay que considerar que la ciudad tiene fuertes pendientes y esto puede influir en dicha elección modal, sin embargo, la literatura nos muestra diversas evidencias de la influencia del factor cultural que privilegia el 


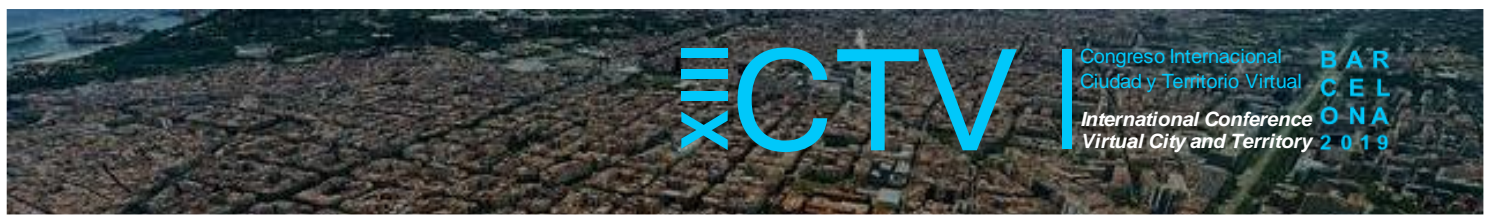

uso del automóvil en las ciudades de América Latina y lo posiciona como uno de los principales retos para la promoción de la movilidad sostenible (Observatorio de Movilidad Urbana para América Latina, 2009; ONU-Hábitat, 2013).

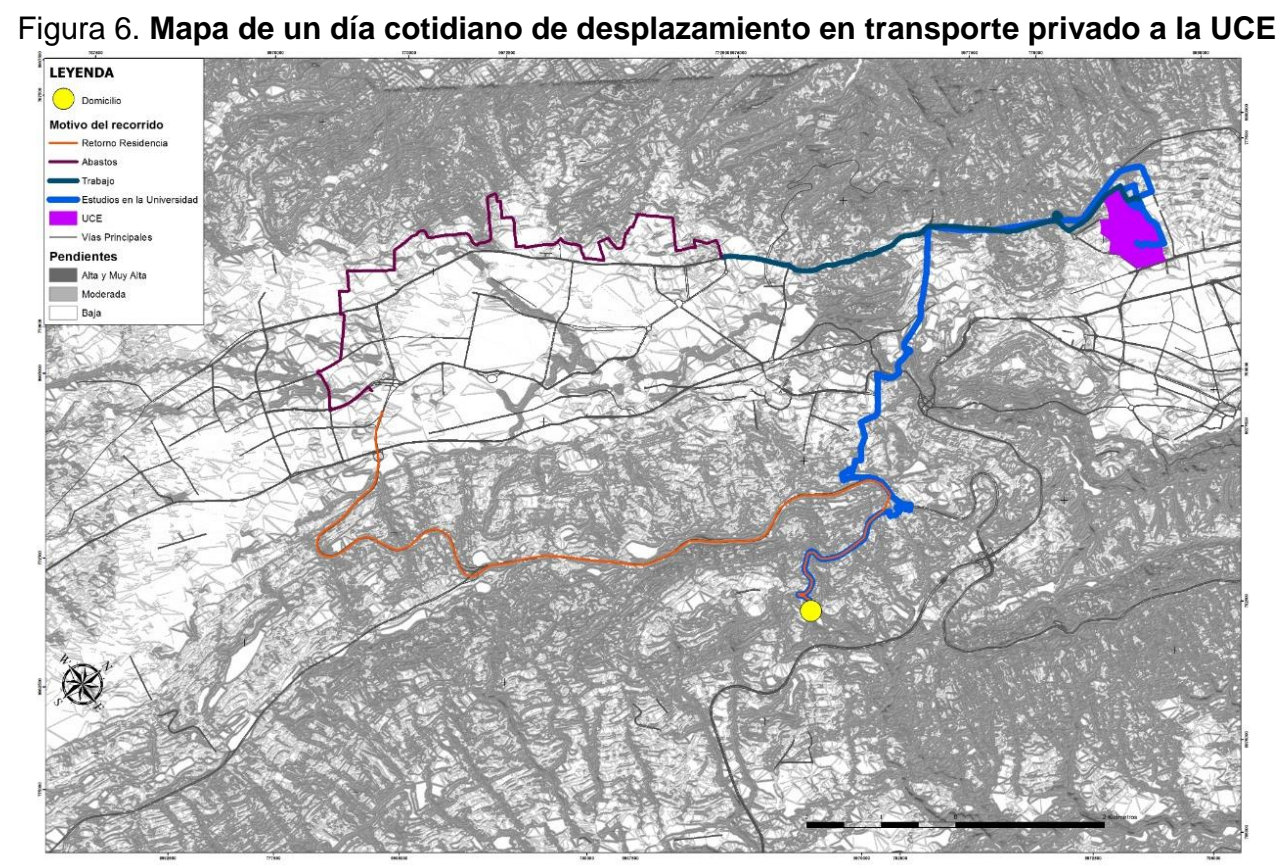

Fuente: Levantamiento de rutas cotidianas de estudiantes de la UCE-FAU

Hay un cierto número de estudiantes que combinan el transporte motorizado privado y el transporte público con aportaciones semejantes en el tiempo de desplazamiento, por lo que no se puede afirmar que uno sea más representativo que el otro (Figura 7).

Figura 7. Mapa de un día cotidiano de desplazamiento compuesto público-privado

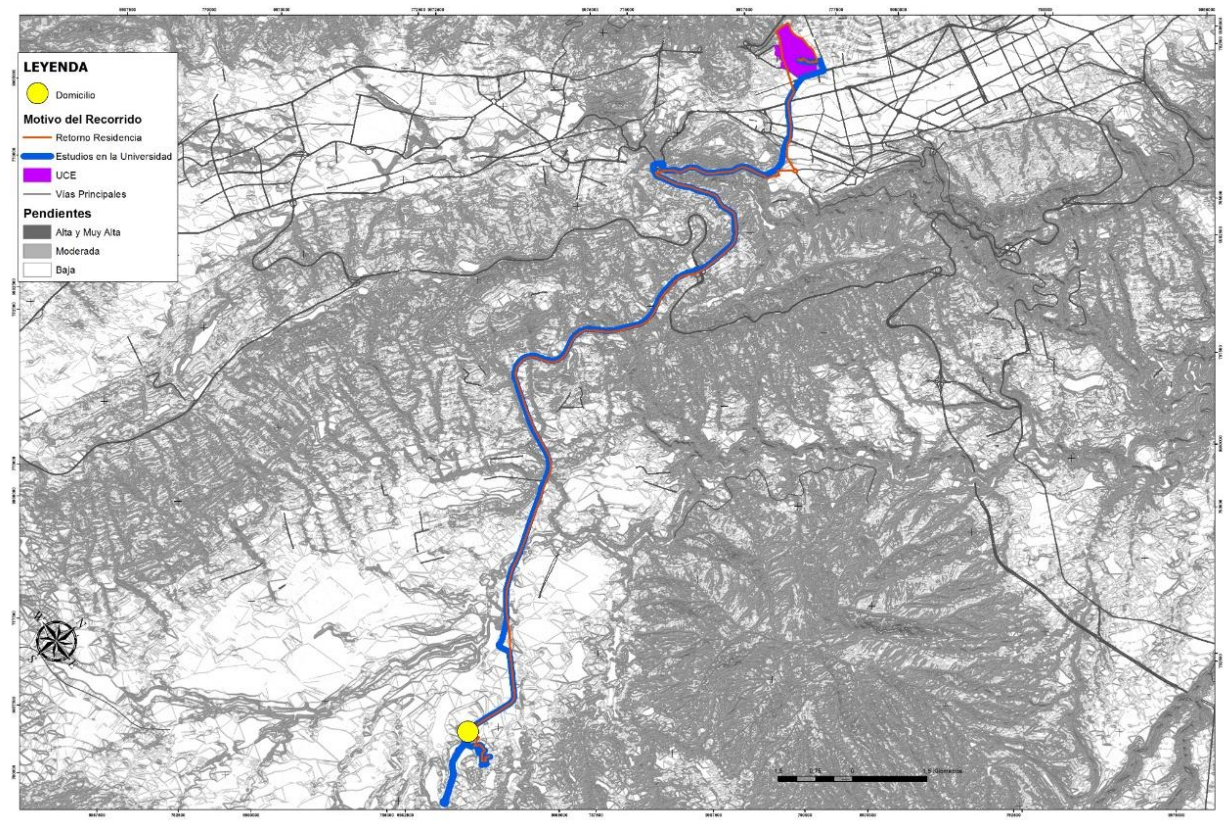

Fuente: Levantamiento de rutas cotidianas de estudiantes de la UCE-FAU 


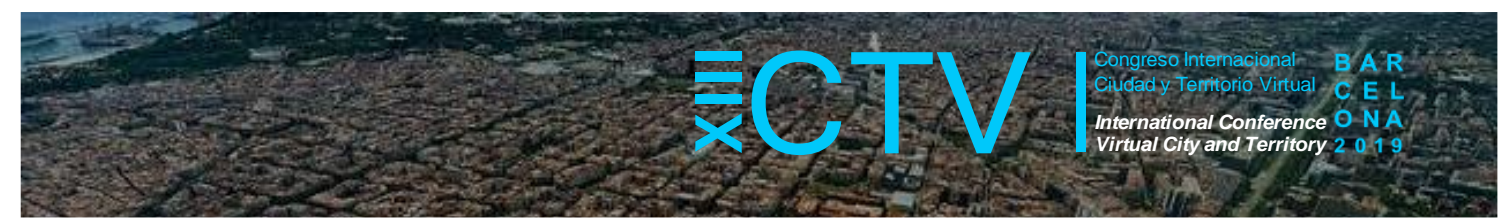

El comportamiento general es que los familiares acerquen a las paradas de transporte público a los estudiantes y que lleguen en este medio a la universidad. Estos estudiantes viven en los límites norte y sur de la ciudad o en los valles, dónde hay menor congestión, situación que puede influir en la primera elección modal, mientras que el transporte público se utiliza en las zonas de mayor centralidad, donde la congestión es tambien mayor en horas punta.

El tiempo promedio del desplazamiento es de 52 minutos, pero existen casos de hasta 76 minutos, de manera que esta organización modal de los desplazamientos contribuye poco para lograr tiempos deseables de hasta 30 minutos, establecidos por organismos internacionales para desplazamientos motorizados (ONU-Habitat, 2016). Se observa que este tipo de despazamietos son generalmente característicos de las estudiantes mujeres que residen en los limites del DMQ y su organización modal mas enfocada en mejorar la seguridad y menos el tiempo del trayecto. Cabe agregar que los estudiantes que tienen actividades laborales, las cuales generalmente están localizadas en el Hipercentro y se desarrollan en horarios vespertinos, hacen menores tiempos de desplazamiento porque se trasladan directamente desde la universidad. Este tipo de desplazamientos son característicos de estudiantes mujeres $\mathrm{y}$ hombres solteros $\mathrm{u}$ hombres casados que asumen el papel de proveedores.

\section{Conclusiones}

Las condiciones socioeconómicas de los estudiantes no son el factor que más influye en la diversidad y frecuencia de las actividades cotidianas que realizan, que se convierten en motivos de desplazamiento y elecciones modales; influye también la accesibilidad de las zonas donde viven y la diversidad funcional para tener posibilidad de aumentar la frecuencia y tipología de actividades y hacer elecciones de desplazamientos no motorizados.

Desde esta perspectiva, la escasa diversidad funcional y accesibilidad de las zonas residenciales localizadas en los límites urbanos del $D M Q$, influyen en el uso de medios motorizados y de largos tiempos de desplazamiento para la realización de la mayor parte de las actividades cotidianas de los estudiantes, incluso más que los aspectos socioeconómicos ya que en estas zonas hay zonas residenciales de nivel medio y medio bajo. Mientras que las zonas residenciales de las estudiantes localizadas en el Hipercentro del DMQ, por su diversidad funcional y accesibilidad, por el contrario, influye positivamente en la diversidad y frecuencia de sus actividades cotidianas con beneficios en su estilo de vida. Esto coincide con una buena parte de la literatura que plantea entre las principales variables para fomentar el uso de medios no motorizados las presencia de altas densidad y mixticidad de uso de suelo (Banister, 2008; Marquet, O. \& Miralles-Guash, C., 2014). La caminabilidad de la ciudad se convierte así en un factor preponderante para compensar los largos tiempos de desplazamiento que los alumnos hacen para estudiar con desplazamientos cortos para desarrollar sus actividades personales relacionadas con e abasto, el ocio y la recreación, el deporte etc. Ryley demostró que los habitantes de Edimburgo compensan los recursos (costo y tiempo) empleados en sus desplazamientos largos, que generalmente son por motivo ocupacional (trabajo, educación) con desplazamientos cortos que realizan a pie o en bicicleta para realizar sus actividades personales (2008).

El transporte público es concebido en el DMQ como un sistema de movilidad orientado a la población de menor capacidad económica y no como un sistema que promueva la sostenibilidad urbana, considerando que se bajo costo fue la principal razón por la cual los estudiantes manifestaron utilizar este medio para llegar a la FAU. En consecuencia, su gestión 


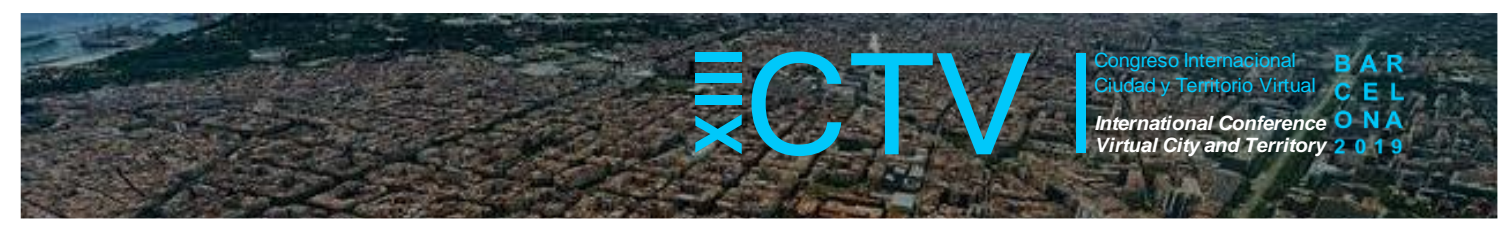

se centra fundamentalmente en la asequibilidad, es decir, que pueda ser financiada por la población de escasos recursos; y se descuidan diversas variables vinculadas con la eficiencia; como la frecuencia de paso, la fiabilidad en el horario de llegada a estaciones o paradas, la seguridad vial y pública; así como variables relacionadas con afectaciones al ambiente como el uso de recursos (energía) y emisión de gases de efecto invernadero. Desde esta perspectiva no se prevé un cambio modal en el que población decida usar el transporte público como un medio eficiente, cómodo, seguro y amigable con el ambiente que están entre los factores que modelan la movilidad urbana sostenible (World Business Council for Sustainable Development 2004).

En la mayoría de los estudios sobre movilidad urbana en ciudades de América Latina se han considerado como variables principales que influyen en los desplazamientos de las personas las relacionadas con los aspectos socio-económicos de la población, lo cual es lógico considerando las condiciones de desigualdad que caracterizan a sus sociedades. Sin embargo, se desprecian otras variables que pueden constituirse en mecanismos para reequilibrar las desigualdades, como es el caso de la diversidad funcional, considerada por una importante cantidad de especialistas reconocidos como la variable principal para fomentar los desplazamientos en medios no motorizados y en particular la caminabilidad (Banister, 2008; Marquet, O. \& Miralles-Guash, C., 2014)

Las características particulares de los casos de la movilidad de las estudiantes mujeres con responsabilidad de hijos de la FAU, nos muestran que hay un componente de género en la organización de los desplazamientos cotidianos, ya que la mayoría de las mujeres de América Latina siguen manteniendo su papel de responsables de los hijos y el hogar, mientras que los hombres siguen manteniendo su papel de proveedores; situación que corrobora la necesidad planteada en distintos estudios sobre el diseño de la ciudad desde las visión de las necesidades de género (Martínez, A. 2012). En este sentido coincidimos con Buckingham en que "El proceso de definir la particular necesidad humana de tener un nivel de vida adecuado debe considerar una multitud de identidades diferentes y cruzadas que habitan en un contexto particular, y cómo su identidad social moldea las formas en que viven y crean el entorno" (2011, pág. 6), y que las mujeres forman parte de estas identidades diferentes con la particularidad de que constituyen la mitad de la población mundial y, por lo general, todos los días tienen violaciones a su derecho colectivo a la ciudad y a la movilidad. Por lo tanto, la perspectiva de genera en la gestión de la movilidad cotidiana podría beneficiar, por lo menos, a la mitad de la población de las ciudades.

Agradecimientos: Esta ponencia es una extensión de la tesis de doctorado de Ma. Isabel Orquera Jácome quien es profesora de la Facultad de Arquitectura de la Universidad Central de Ecuador, institución que, mediante un convenio con la Universidad de Guadalajara, financió el desarrollo del Doctorado en Ciudad, Territorio y Sustentabilidad y otorgó las facilidades a su personal académico para elevar su formación y fomentar la investigación, por lo que esta ponencia no hubiera sido posible sin su apoyo. La directora de la tesis Adriana I. Olivares, profesora también del doctorado mencionado, recibió apoyo del Programa de Apoyo a la Productividad de los Miembros del Sistema Nacional de Investigadores de la Universidad de Guadalajara, donde tienen su adscripción, para asistir a la presentación de la ponencia.

Contribuciones de los autores: La primera autora ha desarrollo los apartados 4 y 5 , el resumen y el procesamiento final y análisis de datos; así como la revisión de estilo y 


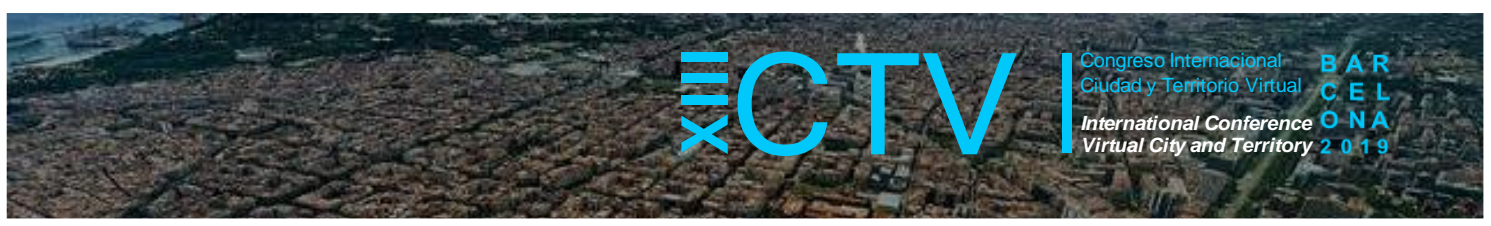

coherencia general del documento. La segunda autora ha desarrollado los apartados 1 al 3 sí como el levantamiento y procesamiento inicial de datos.

Conflicto de Intereses: Los autores declaran que no hay conflicto de intereses.

\section{Bibliografía}

Cuenin, F., \& Silva, M. (2010). Identificación y Fortalecimiento de Centralidades Urbanas, El Caso de Quito. Inter-American Development Bank.

D’Ercole, R., \& Metzger, P. (2004). La vulnerabilidad del Distrito Metropolitano de Quito. Quito: IMQ - IRD.

Galaxy, M. (02 de noviembre de 2017). Maps Galaxy. Obtenido de http://www.gifex.com/fullsize/2011-10-25-14669/Parroquias-de-Quito-2001.html

IMPU. (2018). Quito, visión 2040 y su nuevo modelo de ciudad. Quito: MDQ.

IMQ Dirección de Planificación. (1992). Estructura espacial metropolitana: criterios y problemas críticos. Quito: El Conejo.

Martínez Elvir, Amanda (2012) Mujer y movilidad urbana. La planeación de la ciudad desde la perspectiva de género.

Miralles-Guash, (2008). The social use of time in the Metropolitan region of Barcelona. Barcelona: Institut d'Estudis Regionals i Metropolitans de Barcelona.

Miralles-Guash, C. (2011) "Dinámicas metropolitanas y tiempos de la movilidad. La región metropolitana de Barcelona, como ejemplo". Anales de Geografía vol. 31, núm. 1, 125-145.

Miralles-Guash, C. \& Oriol Marquet Sarda (2012) "Dinámicas de proximidad en ciudades multifuncionales". Ciudad y Territorio. Estudios Territoriales. № 177, 2013, págs 501-5010.

Marquet, O., \& Miralles-Guasch, C. (2014) "The Walkable city and the importance of the proximity environments for Barcelona's everyday mobility. J. Cities, DOI http://dx.doi.org/10.1016/i.cities.2014.10.012

Observatorio de Movilidad Urbana para América Latina (2007-2011), Banco de Desarrollo de

América Latina, Comisión Andina de Fomento. Recuperado de https://www.caf.com/es/conocimiento/datos/observatorio-de-movilidad-urbana/

Observatorio de Movilidad Urbana para América Latina. Información para mejores políticas y mejores ciudades, 2009.

ONU-Habitat (2016) Measurement of City Prosperity. Methodology and Metadata.

ONU-Habitat (2016) Planificacion y Diseño de una movilidad urbana sostenible: orientaciones para politicas. Informe Mundial sobre asentamientos Humanos 2013.

Rosas Bolbotín, Patricio y Liliana Salazar Arredondo (2015) Violencia de género en el transporte público. Una regulación pendiente, CEPAL.

Ryley, T. J. (2008). The propensity for motorists to walk for short trips: Evidence from West Edinburgh. Transportation Research Part A: Policy and Practice, 42(4), 620-628.

SENESCYT. (15 de octubre de 2017). Geoportal SNIESE. Recuperado de http://www.senescyt.gob.ec/visorgeografico/

World Business Council for Sustainable Development (2004), Mobility 2030, Meeting de challenges to sustainability. 